\title{
Dosimetric evaluation of Tomotherapy and four-box field conformal radiotherapy in locally advanced rectal cancer
}

\author{
Mina Yu, MD', Hong Seok Jang, MD², Dong Min Jeon, $\mathrm{MS}^{2}$, Geum Seong Cheon, BA², \\ Hyo Chun Lee, MD1', Mi Joo Chung, MD1, Sung Hwan Kim, MD', Jong Hoon Lee, MD¹ \\ 'Department of Radiation Oncology, St. Vincent's Hospital, The Catholic University of Korea College of Medicine, Suwon; \\ ${ }^{2}$ Department of Radiation Oncology, Seoul St. Mary's Hospital, The Catholic University of Korea College of Medicine, Seoul, Korea
}

Purpose: To report the results of dosimetric comparison between intensity-modulated radiotherapy (IMRT) using Tomotherapy and four-box field conformal radiotherapy (CRT) for pelvic irradiation of locally advanced rectal cancer.

Materials and Methods: Twelve patients with locally advanced rectal cancer who received a short course preoperative chemoradiotherapy (25 Gy in 5 fractions) on the pelvis using Tomotherapy, between July 2010 and December 2010, were selected. Using their simulation computed tomography scans, Tomotherapy and four-box field CRT plans with the same dose schedule were evaluated, and dosimetric parameters of the two plans were compared. For the comparison of target coverage, we analyzed the mean dose, $V_{n \text { Gy }}, D_{\text {min, }} D_{\text {max }}$ radical dose homogeneity index $(r \mathrm{DHI})$, and radiation conformity index $(\mathrm{RCl})$. For the comparison of organs at risk (OAR), we analyzed the mean dose.

Results: Tomotherapy showed a significantly higher mean target dose than four-box field CRT ( $p=0.001$ ). But, $V_{26.25}$ Gy and $V_{27.5}$ Gy were not significantly different between the two modalities. Tomotherapy showed higher $\mathrm{D}_{\max }$ and lower $\mathrm{D}_{\min }$. The Tomotherapy plan had a lower rDHI than four-box field CRT ( $p=0.000)$. Tomotherapy showed better RCI than four-box field CRT $(p=0.007)$. For OAR, the mean irradiated dose was significantly lower in Tomotherapy than four-box field CRT.

Conclusion: In locally advanced rectal cancer, Tomotherapy delivers a higher conformal radiation dose to the target and reduces the irradiated dose to OAR than four-box field CRT.

Keywords: Rectal neoplasms, Helical tomotherapy, Conformal radiotherapy, Radiation dosimetry, Preoperative procedure

\section{Introduction}

Cancers of the colon and rectum are the third most common cancer diagnosed in men and women worldwide. In Korea, colorectal cancers are estimated to have a high rate of incidence (2nd highest rate in males and 3rd highest rate in females), and to be common causes of cancer-related deaths (3rd most common cause in males and 2nd most common cause in females) in the current year [1]. Rectal cancers account for about one-third of these colorectal cancers [2].

Recently, preoperative chemoradiotherapy has been generally used in the management of locally advanced rectal cancer on the basis of several benefits proven by clinical studies, in the aspect of better locoregional tumor control, reduced toxicity of normal organs, and an increased chance of preserving the anal sphincter, when compared with postoperative

Received 5 September 2013, Revised 14 October 2013, Accepted 22 October 2013.

Correspondence: Jong Hoon Lee, MD, Department of Radiation Oncology, St. Vincent's Hospital, 93 Jungbu-daero, Paldalgu, Suwon 442-723, Korea. Tel: +82-31-249-7560, Fax: +82-31-257-3734, E-mail: koppul@catholic.ac.kr

(c) This is an Open Access article distributed under the terms of the Creative Commons Attribution Non-Commercial License (http://creativecommons.org/ licenses/by-nc/3.0/) which permits unrestricted non-commercial use, distribution, and reproduction in any medium, provided the original work is properly cited. 
chemoradiotherapy [2-5]. There have been two kinds of schedules in preoperative radiotherapy. One is a long-course treatment using conventional doses of 1.8 to 2 Gy per fraction, with a total dose of 45 to 50 Gy over 5 to 6 weeks, and the other is a short-course treatment using 5 Gy per fraction, with a total dose of 25 Gy during 1 week. In Europe, especially, a short-course radiotherapy (SC-RT) is preferred, because of its convenience, simplicity, and lower acute toxicity than longcourse RT (LC-RT) [4,6-14]. But, SC-RT could significantly increase late complications like anastomotic stricture or small bowel obstruction $[6,9,15-19]$.

Therefore, concerning these late complications, KROG 1001, a phase II multicenter study (preoperative short-course chemoradiotherapy followed by delayed surgery for rectal cancer), enrolled patients receiving RT using Tomotherapy to minimize irradiation to normal tissues. This study was performed for the dosimetric comparison of Tomotherapy and four-box field conformal radiotherapy (CRT) planning for pelvic irradiation of patients with locally advanced rectal cancer.

\section{Materials and Methods}

\section{Patients}

We reviewed 12 patients, who were involved in KROG 10-01, with histologically confirmed adenocarcinoma of the rectum, clinical stage $\mathrm{T} 3$ or resectable $\mathrm{T} 4$. They received primary chemoradiotherapy at Seoul St. Mary's Hospital between July 7, 2010 and December 31, 2010. Their tumors were all located within $8 \mathrm{~cm}$ above the anal verge. Patients were 18 years old or older, and their Eastern Cooperative Oncology Group performance status was 0 to 2 . They had adequate bone marrow, kidney, and liver functions. Patients with distant metastases, lateral pelvic node of $\geq 0.5 \mathrm{~cm}$ sized, or another primary cancer history were excluded. Moreover, patients who were not appropriate for concurrent chemoradiotherapy were also excluded.

All 12 patients received Tomotherapy (TomoTherapy Inc., Madison, WI, USA) actually, but we also planned four-box field CRT for dosimetric comparison of the two plans. The Institutional Review Board approved this study (KC10EIMS0025).

\section{Intensity-modulated radiotherapy (IMRT) planning using Tomotherapy}

The prescribed dose was 25 Gy in 5 fractions to planning target volume (PTV). Computed tomography (CT) simulation was done in all patients in prone position using a belly board. Contouring the target volumes and normal organs for helical Tomotherapy is described in the KROG 10-01 report [20]. These target volumes were also used for the CRT planning. Clinical target volume (CTV) included gross tumor and mesorectum, presacral
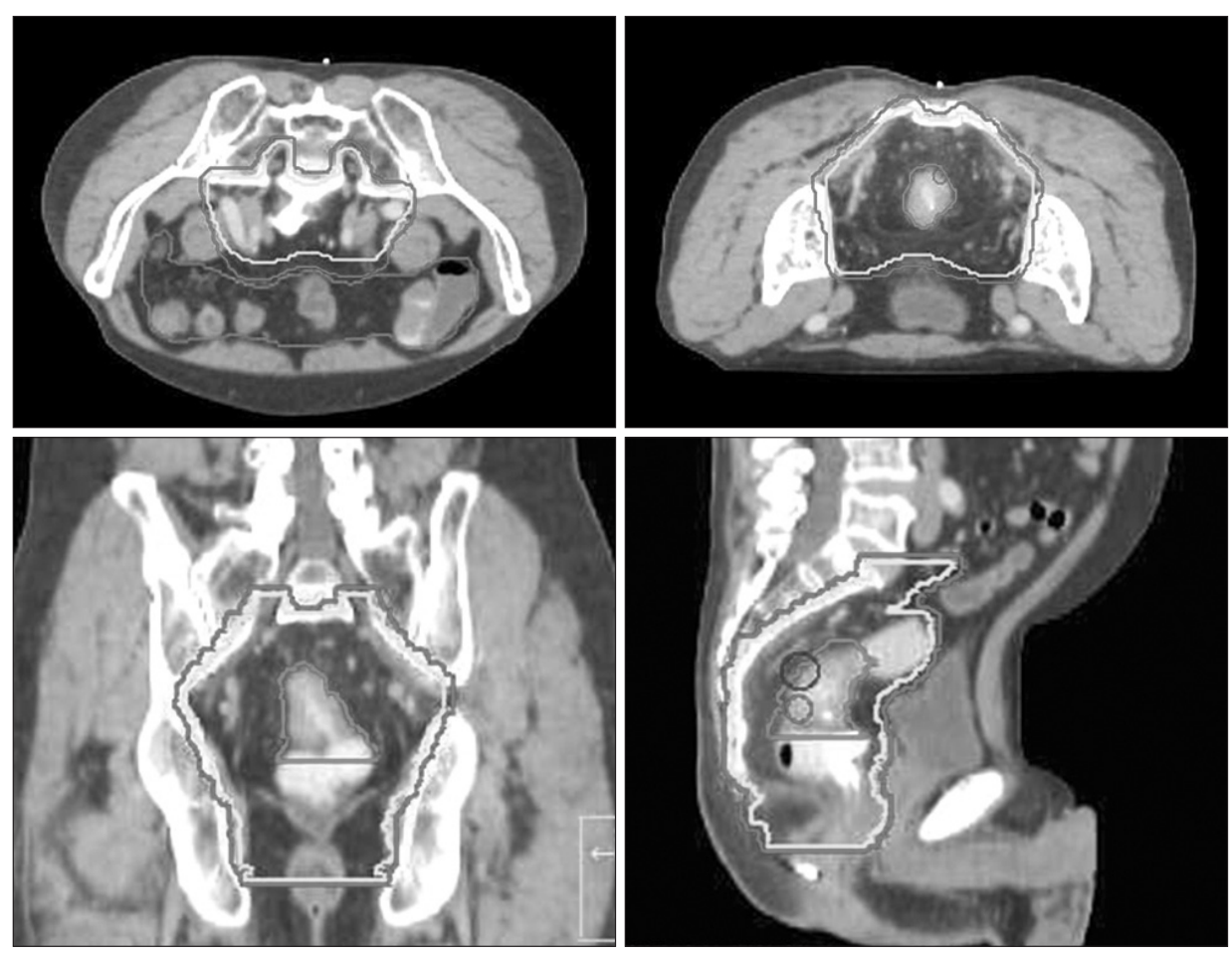

Fig. 1. Contours of the planning target volume (PTV) and bowel. The outermost line is PTV. The bowel is contoured as the peritoneal space. 
space, the entire sacral hollow, and regional lymphatics (Fig. 1). PTV was generated with at least $3 \mathrm{~mm}$ margin from the CTV. We defined bowel as the peritoneal space above sigmoid colon. The IMRT planning goals were delivering at least 93\% of the prescribed dose to 98\% of the PTV, while delivering 105\% of the prescribed dose to below 10\%, 110\% of the prescribed dose to below $5 \%$, and not delivering $\geq 115 \%$ of the prescribed dose to the PTV.

We kept the maximal irradiation dose of the bladder under $24 \mathrm{~Gy}$, bowel under $25 \mathrm{~Gy}$, and femur heads under $20 \mathrm{~Gy}$ and kept the relative volumes of the above 3 organs at risk (OARs), irradiated $\geq 11 \mathrm{~Gy}, \geq 3 \mathrm{~Gy}$, and $\geq 15 \mathrm{~Gy}$, respectively, under 50\%. We did our best to minimize the irradiated volume of the bowel and the radiation dose to other normal organs.

Actually, every patient was treated by the Tomotherapy HiArt system (TomoTherapy Inc.) using 6-MV photons. We used $2.5 \mathrm{~cm}$ of fan beam thickness (FBT) to reduce the penumbra in the longitudinal direction (when compared to $5 \mathrm{~cm}$ of FBT) and overall treatment time (when compared to $1 \mathrm{~cm}$ of FBT). Pitch was 0.3. The modulation factor ranged from 2 to 2.4, depending on homogeneity and conformality. Inverse planning using numerical dose-volume constraints was done.

\section{Four-box field conformal radiotherapy planning}

No one was treated with CRT, but we planned it for comparison. CRT plans were done using the Pinnacle ${ }^{3}$ treatment planning system (Philips Radiation Oncology Systems, Fitchburg, WI, USA). The isocenter was at the center of the widest pelvic inlet. Anterior-posterior/posterior-anterior (6 MV) and bilateral ports (15 MV) were used. The superior border of the PTV was lumbosacral junction, and the inferior border of the PTV was at least $3 \mathrm{~cm}$ below the lowest level of the gross lesion. The superior and the inferior border of the field was $1 \mathrm{~cm}$ apart from the contoured PTV, and lateral border was also $1 \mathrm{~cm}$ apart from the PTV or $1.5 \mathrm{~cm}$ beyond the widest pelvic inlet. The lateral fields extended from the posterior edge of the symphysis pubis or $1 \mathrm{~cm}$ anterior of the PTV to $1 \mathrm{~cm}$ posterior to the sacral body or $1 \mathrm{~cm}$ posterior to the PTV, but not beyond the skin of the natal cleft. Block margin was $1 \mathrm{~cm}$ from the PTV (Fig. 2).

\section{Evaluation and comparison of treatment plans}

We computed several parameters to compare the two different plans. The mean dose, the volume irradiated 93\%, 105\%, and $110 \%$ of the prescribed dose, the minimum dose $\left(D_{\min }\right)$, and maximum dose $\left(D_{\max }\right)$ were analyzed for the PTV. For OAR like the bowel and bladder, both femur heads, the mean doses were analyzed. And the volume irradiated with $20 \mathrm{~Gy}\left(\mathrm{~V}_{20}\right.$ ${ }_{\text {Gy }}$ ) in each technique was also analyzed to show the volume that received intermediate radiation dose. We also calculated the radical dose homogeneity index ( $\mathrm{rDHI})$ and radiation conformity index (RCI) for the PTV. The rDHI was defined as the ratio of the minimal dose to the maximal dose in the PTV $\left(r D H I=D_{\min } / D_{\text {max }}\right.$ in PTV) [21]. The $R C l$ was defined as the ratio of the whole volume of PTV to the irradiated volume at 95\% of the prescribed dose $\left(\mathrm{RCl}=\mathrm{V}_{\text {PTV }} / \mathrm{V}_{95 \%}\right)$ [22].

For all dosimetric parameters of Tomotherapy and four-box field CRT, normality tests were done. As a result, mean doses of the PTV and OARs, which have shown normal distributions, were compared using t-test, and the other parameters were compared using Mann-Whitney test. A difference was considered statistically significant at the $p<0.05$ level.
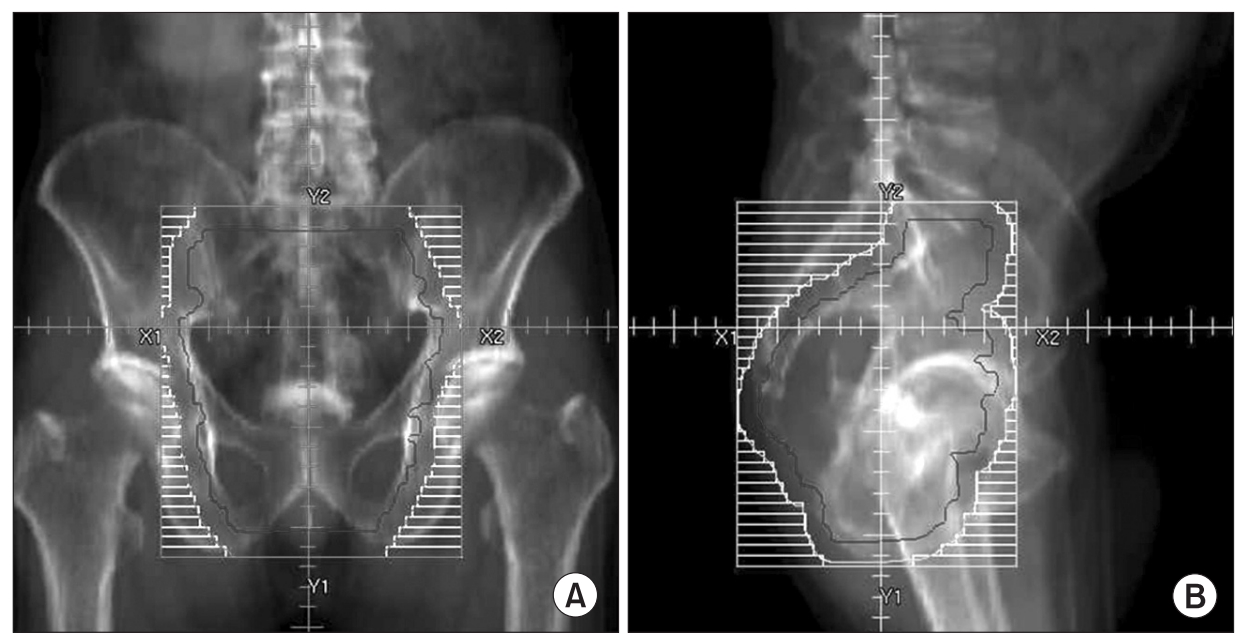

Fig. 2. Beam's eye view of the four-box conformal radiotherapy planning. (A) Anterio-posterior field and (B) right lateral field. Black line is planning target volume (PTV), and $1 \mathrm{~cm}$ of uniform multileaf collimator margin from PTV was generated all directionally. 


\section{Results}

\section{Dose distribution of the target}

The median volume of the contoured PTV in the 12 patients was 1,028.2 $\mathrm{mL}$ (range, 796.4 to $1,231.57 \mathrm{~mL}$ ). The prescription goal of the PTV was irradiating at least 23.25 Gy (93\% of the prescribed dose) to $98 \%$ of the PTV. In all enrolled patients who received Tomotherapy, the goal was satisfied $\left(V_{23.25 G y}=\right.$ $98.81 \% \pm 0.24 \%) . V_{23.25}$ Gy value was significantly higher in the Tomotherapy arm than the CRT arm $(98.81 \% \pm 0.24 \%$ vs. $95.55 \% \pm 4.32 \%, p=0.007)$.

Table 1 lists the dosimetric parameters of the Tomotherapy and CRT plans, and Fig. 3 shows the dose-volume histograms of the PTV with two different techniques. The mean PTV dose $(25.08 \pm 0.24$ vs. $25.40 \pm 0.15, p=0.001)$ and the $D_{\max }$ value $(26.49 \pm 0.33$ vs. $27.43 \pm 0.77, p=0.000)$ were significantly lower in the CRT arm than the Tomotherapy arm. The $D_{\text {min }}$ value was significantly higher in the CRT arm than the Tomotherapy $\operatorname{arm}(21.71 \pm 2.81$ vs. $18.76 \pm 1.93, p=0.000)$. However, the $V_{26.25 \text { Gy }}\left(105 \%\right.$ of the prescribed dose) value and $V_{27.5 \mathrm{~Gy}}(110 \%$ of the prescribed dose) were not significantly different between the two modalities. (CRT vs. Tomotherapy; $V_{26.25 \text { Gy }}: 1.85 \pm$ 3.11 vs. $1.46 \pm 1.82, p=0.378 ; V_{27.5}$ Gy $: 0.00 \pm 0.00$ vs. $0.05 \pm$ $0.13, p=0.514)$. The $r D H I$ value was significantly lower in the Tomotherapy plan than the CRT plan $(0.68 \pm 0.06$ vs. $0.81 \pm$ $0.10, p=0.000$ ). The $R C l$ value was significantly better in the Tomotherapy plan than the CRT plan $(1.01 \pm 0.00$ vs. $1.04 \pm$

Table 1. Evaluated dosimetric parameters of PTV for comparison of two different plans

\begin{tabular}{lrcc}
\hline \multicolumn{1}{c}{ Value } & Tomotherapy & Four-box field CRT & $p$-value \\
\hline Mean dose $(G y)$ & $25.40 \pm 0.15$ & $25.08 \pm 0.24$ & 0.001 \\
$V_{23.25 \text { Gy }}(\%)$ & $98.81 \pm 0.24$ & $95.55 \pm 4.32$ & 0.007 \\
$V_{26.25 \text { Gy }}(\%)$ & $1.46 \pm 1.82$ & $1.85 \pm 3.11$ & 0.378 \\
$V_{27.5 \mathrm{~Gy}}(\%)$ & $0.05 \pm 0.13$ & 0 & 0.514 \\
$D_{\text {min }}(\mathrm{Gy})$ & $18.76 \pm 1.93$ & $21.71 \pm 2.81$ & 0.000 \\
$\mathrm{D}_{\text {max }}(\mathrm{Gy})$ & $27.43 \pm 0.77$ & $26.49 \pm 0.33$ & 0.000 \\
$\mathrm{RCl}$ & $1.01 \pm 0.00$ & $1.04 \pm 0.04$ & 0.007 \\
$\mathrm{rDHI}$ & $0.68 \pm 0.06$ & $0.81 \pm 0.10$ & 0.000 \\
\hline
\end{tabular}

Values are presented as mean \pm standard deviation.

PTV, planning target volume; $C R T$, conformal radiotherapy; $V_{n} G_{y}$ percentage of the volume receiving radiation $\geq n G y ; D_{\text {min }}$ minimum dose to the PTV; $D_{\text {max }}$ maximum dose to the PTV; $R C$, radiation conformity index $\left(V_{P T V} / V_{95 \%}\right.$ of prescribed dose), rDHI, radical dose homogeneity index $\left(D_{\min } / D_{\max }\right.$ in $\left.P T V \leq 1\right)$.

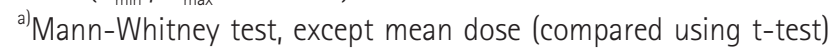
which showed normal distribution.
$0.04, p=0.007)$. Fig. 4 shows the CT slides that represent the 95\% isodose distributions for the two modalities. Tomotherapy planning showed better dose conformation than CRT planning.

\section{Dose distribution of the organs at risk}

Table 2 and Fig. 5 show the mean dose and the DVHs for the bowel, bladder, and both femur heads. Tomotherapy planning enabled all OARs to be irradiated with a significantly lower mean dose $(p<0.05)$, and showed a significantly lower $V_{20 G y}$ of the bowel, the bladder, and both femur heads $(p=0.000)$ than CRT.

\section{Discussion and Conclusion}

Today, patients with locally advanced rectal cancer typically receive conventionally fractionated preoperative chemoradiotherapy with the 3- or 4-portal technique. Since the total irradiated dose to the rectum is $50.4 \mathrm{~Gy}$ in 28 fractions, even if the small bowel is included in the irradiation field, the incidence of severe toxicity over grade 3 is acceptable.

In Europe, SC-RT (25 Gy in 5 fractions) has been commonly used in rectal cancer patients, and it has shown favorable results in disease control and a lower incidence of acute complications $[7,10]$. TROG 01.04, a randomized trial which compared preoperative SC-RT versus long-course chemoradiotherapy (LC-CRT), reported little difference in incidence of grade 3 to 4 severe late complications ( $5.8 \%$ vs. $8.2 \%, p=0.53$ ) and incidence of severe late bowel toxicities ( $3.2 \%$ vs. $5.1 \%, p=0.53$ ) [10]. Their median follow-up time was 5.9 years (range, 3 to 7.8 years). Another randomized trial, Polish trial showed similar conclusions on late complications [7]. In this study, the overall

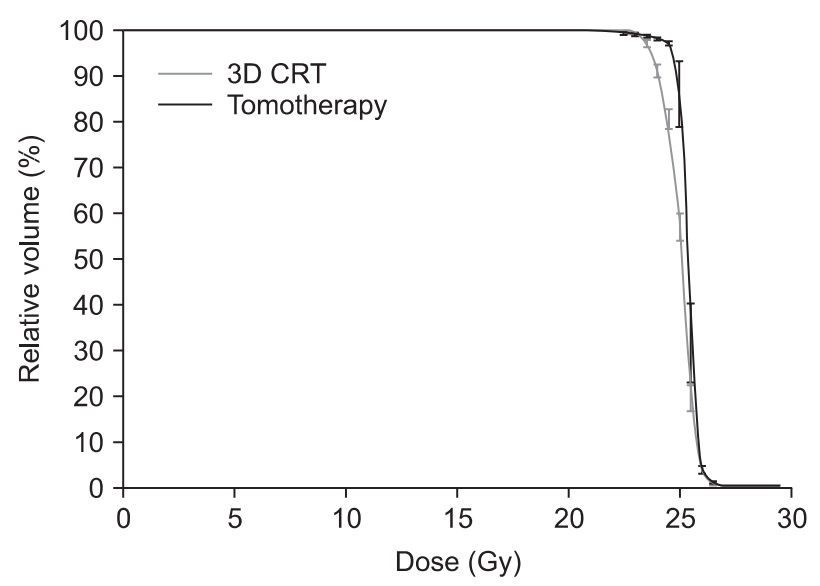

Fig. 3. Dose-volume histograms of the planning target volume. 3D CRT, 3-dimensional conformal radiotherapy. 

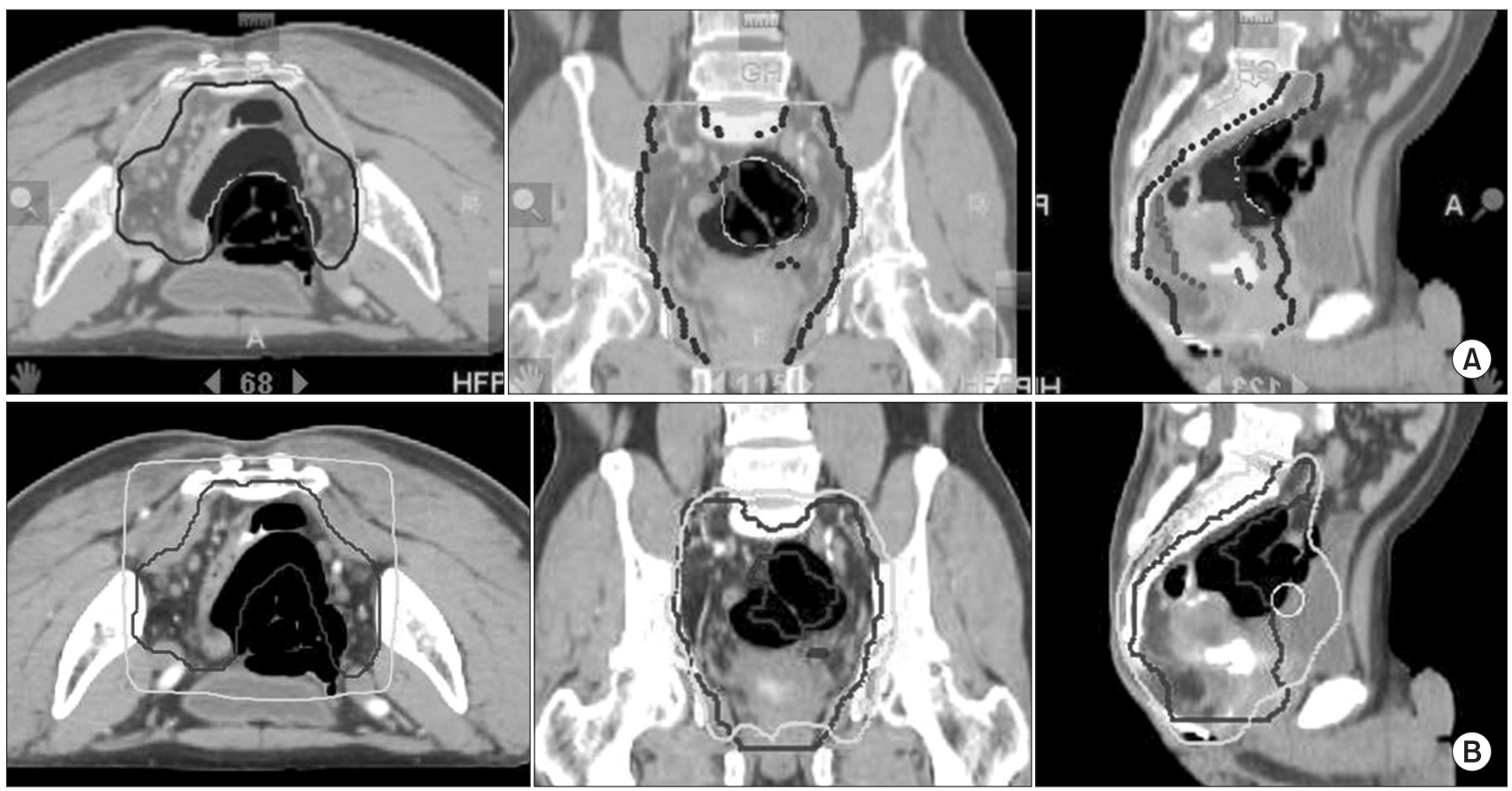

Fig. 4. Dose distribution of Tomotherapy (A) and 3-dimensional radiotherapy (B). We can see that isodose line in Tomotherapy planning is shaped into geometrical configuration of the planning target volume (PTV), and the irradiated volume of the bowel and the bladder at high dose is smaller in Tomotherapy than in 3-dimensional radiotherapy. Gray line represents 95\% isodose line and black contour is PTV.

Table 2. Analysis of normal tissue irradiation dose and $V_{20 \text { Gy }}$ between Tomotherapy and four-box field conformal radiotherapy (CRT)

\begin{tabular}{|c|c|c|c|c|c|c|}
\hline Normal tissue & $\begin{array}{c}\text { Tomotherapy } \\
\text { mean (Gy) }\end{array}$ & $\begin{array}{c}\text { Four-box field CRT } \\
\text { mean (Gy) }\end{array}$ & p-value & $\begin{array}{c}\text { Tomotherapy } \\
V_{20 \text { Gy }}(\mathrm{mL})\end{array}$ & $\begin{array}{c}\text { Four-box field CRT } \\
\qquad V_{20 \mathrm{~Gy}}(\mathrm{~mL})\end{array}$ & $p$-value ${ }^{a)}$ \\
\hline Bowel & $8.26 \pm 2.51$ & $12.75 \pm 4.39$ & 0.007 & $4.98 \pm 7.87$ & $25.76 \pm 19.85$ & 0.000 \\
\hline Bladder & $13.66 \pm 1.52$ & $21.41 \pm 1.84$ & 0.001 & $17.18 \pm 7.31$ & $65.86 \pm 17.33$ & 0.000 \\
\hline Right femur head & $10.31 \pm 1.61$ & $14.34 \pm 1.34$ & 0.001 & $0.25 \pm 0.87$ & $4.21 \pm 4.12$ & 0.000 \\
\hline Left femur head & $10.80 \pm 1.75$ & $14.94 \pm 1.44$ & 0.001 & $0.07 \pm 0.23$ & $3.22 \pm 3.42$ & 0.000 \\
\hline
\end{tabular}

Values are presented as mean \pm standard deviation.

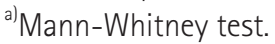

incidence of late toxicities in SC-RT vs. LC-CRT was $28.3 \%$ vs. $27 \%(p=0.810)$, and the incidence of severe late toxicities was $10.1 \%$ vs. $7.1 \%$ ( $p=0.360$ ). However, they reported the relative risk of severe late toxicity in the SC-RT compared with the LCCRT as 1.43. They did not analyze statistical significance in the incidence of severe late bowel toxicity, but, it was 5.1\% in the SC-RT group and 1.4\% in the LC-CRT. Their median follow-up time was 4 years (range, 2.6 to 5.8 years).

On the other hand, in the study which did long-term quality of life assessment of the patients in Stockholm I and II trial (mean follow-up time, 15 years; range, 9 to 21 years), 69\% of the 65 survivors treated with SC-RT had any adverse event, and the small bowel obstruction amounted to $29 \%$ [18].
Furthermore, Dutch trial reported 51\% of fecal incontinence rate in patients treated with SC-RT [17], and Swedish trial reported that the relative risk of bowel obstruction was increased after approximately 8 years [19]. Guckenberger et al. [9] reported the incidence of severe late bowel toxicity of SCRT as up to over $12 \%$ compared to LC-RT 3\%. Thus, drawing a conclusion that SC-RT has acceptable late complications is hasty and great efforts to reduce the irradiation dose to the OARs should be made.

IMRT delivers at least an equal or higher dose to the target and a lesser dose to the OAR than 3-dimensional RT. Thus, it becomes abreast of 3-dimensional RT in disease control and can reduce radiation-induced complications. Several studies 
A

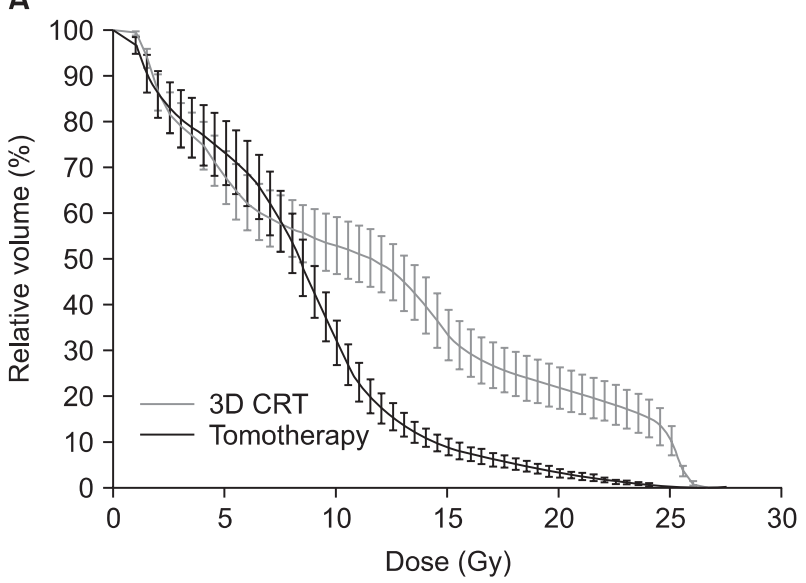

C

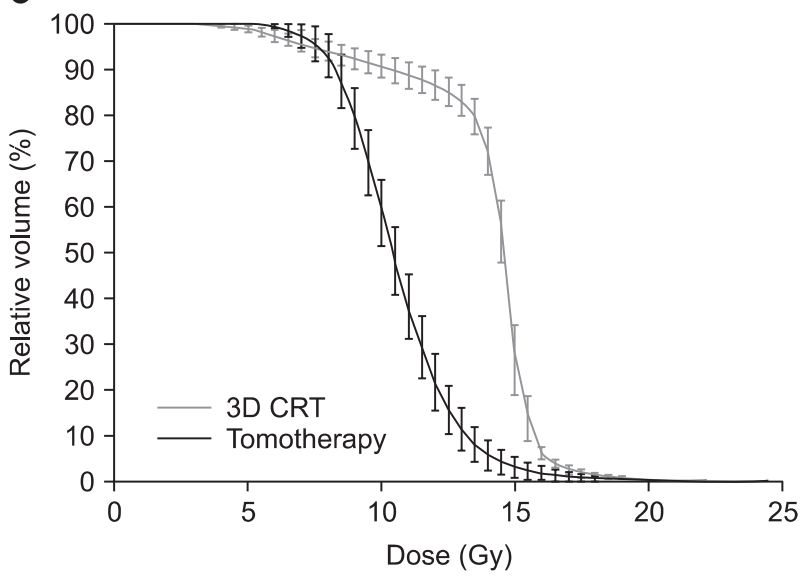

B

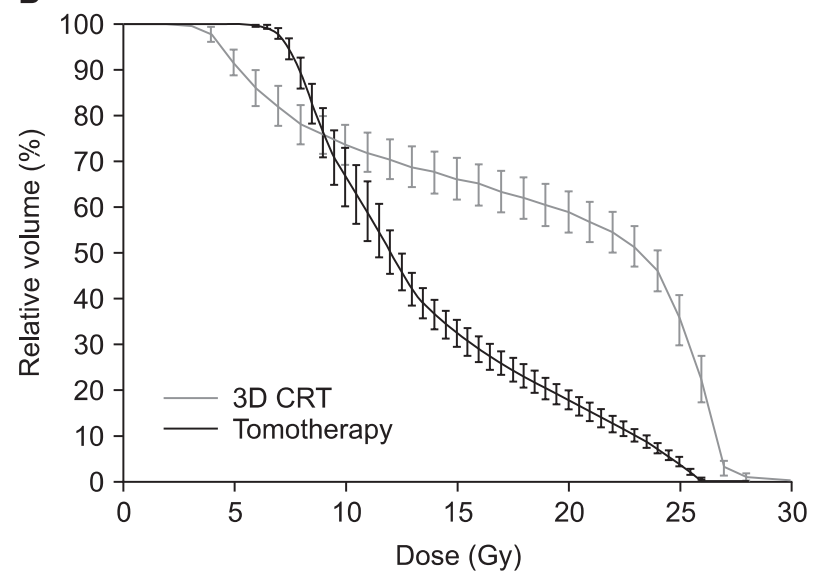

D

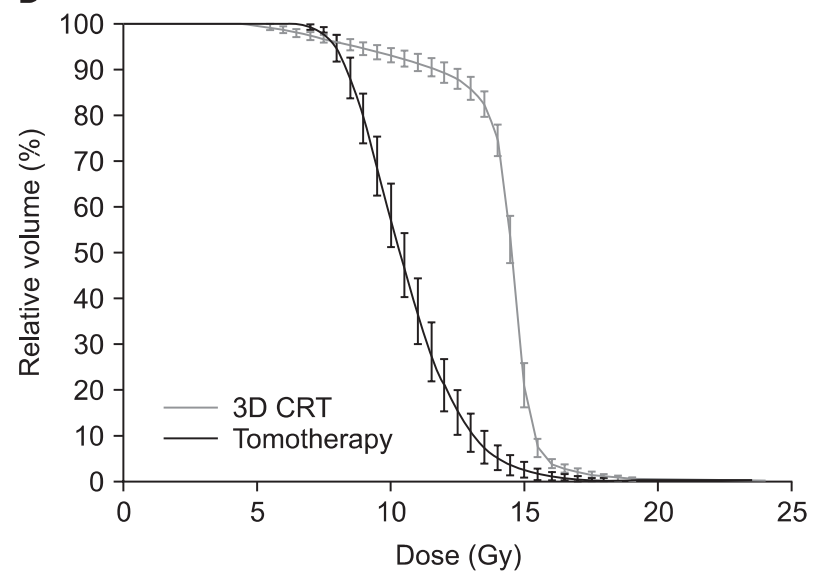

Fig. 5. Dose-volume histograms of the organs at risk. (A) Bowel, (B) bladder, (C) right femur head, and (D) left femur head. 3D CRT, 3-dimensional conformal radiotherapy.

investigating prostate cancer, gynecologic cancer, and anal cancer have proven these advantages [23-26]. However, in rectal cancer, studies which show the dosimetric comparison between IMRT and 3-dimensional RT are rare. Arbea et al. [27] reported on the dosimetric comparison of IMRT and 3-dimensional RT in 2010. They performed a linac-based IMRT, and the IMRT was applied to the conventional schedule. Mean $D_{95}$ (dose that covers $95 \%$ of the PTV), homogeneity index $(\mathrm{HI})$, conformity index $(\mathrm{Cl})$, and $\mathrm{D}_{5}$ (dose that covers $5 \%$ of the region of interest), $V_{40}$ Gy of OAR (small bowel, bladder) were evaluated. IMRT showed a significantly lower mean $D_{95}$ of the PTV (46.9 Gy vs. $47.5 \mathrm{~Gy}, \mathrm{p}<0.05)$ and better conformality (0.8 vs. 0.6, $p<0.05$ ) than 3 -dimensional RT at the expense of dose homogeneity. The researchers pointed out that there was a trade-off between target coverage and normal organ avoidance, and dose inhomogeneity within the target occurred in IMRT planning. But, the volume of the underdosage in PTV was not so great, and the position of the cold spot was in the area where the tumor was not likely to invade, and therefore, tumor control might not be reduced. In OAR, IMRT showed a vast reduction of the mean $\mathrm{V}_{40 \mathrm{~Gy}}$ and $\mathrm{D}_{5}$.

Our study showed directly whether Tomotherapy had dosimetric merits or not when it was applied with a shortcourse schedule to patients with rectal cancer. We previously published a study on the dosimetric comparison between Tomotherapy and 3-portal CRT. In that study, we found that Tomotherapy showed a significantly higher mean dose of PTV (25.58 \pm 0.35 vs. $25.19 \pm 0.74, p=0.043)$ [28]. rDHI was significantly lower in Tomotherapy than 3-dimensional RT like the study by Arbea et al. [27], and a comparison of the irradiation dose in OAR showed that Tomotherapy could largely reduce the mean dose and the volume of high dose exposure. In this paper now, we compared IMRT using Tomotherapy and four-box field CRT. Like our previous study, Tomotherapy showed a higher mean dose of PTV and a more inhomogeneous dose distribution. But unlike the previous 
study, dose conformality of Tomotherapy was better than that of four-box field CRT. In addition, the irradiated mean dose of the normal organs was about two-thirds of that of 3-dimensional RT. Especially we compared $V_{20 \text { Gy }}$ of OARs in the two plans. That is to say, we intended to show the volume that received intermediate radiation dose. All compared $V_{20}$ gy of OARs in Tomotherapy were significantly lower than in 3-dimensional RT.

Therefore, we can expect that in rectal cancer, Tomotherapy enables a favorable outcome in disease control, and also provides a chance to reduce complications of normal tissues. However, this study was a retrospective analysis with a small number of cases, and did not reflect the actual clinical results. In the future, we have to find the optimal short-course regimen, and with a proper schedule, Tomotherapy planning needs to be analyzed with the clinical results.

\section{Conflict of Interest}

No potential conflict of interest relevant to this article was reported.

\section{Acknowledgments}

This work was supported by Young Investigator Fund of the Korean Society of Radiation Oncology (KOSRO).

\section{References}

1. Jung KW, Won YJ, Kong HJ, Oh CM, Seo HG, Lee JS. Prediction of cancer incidence and mortality in Korea, 2013. Cancer Res Treat 2013;45:15-21.

2. Lee JH, Kim SH, Kim JG, Cho HM, Shim BY. Preoperative chemoradiotherapy (CRT) followed by laparoscopic surgery for rectal cancer: predictors of the tumor response and the longterm oncologic outcomes. Int J Radiat Oncol Biol Phys 2011; $81: 431-8$

3. Lee JH, Jang HS, Kim JG, et al. Lymphovascular invasion is a significant prognosticator in rectal cancer patients who receive preoperative chemoradiotherapy followed by total mesorectal excision. Ann Surg Oncol 2012;19:1213-21.

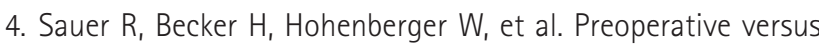
postoperative chemoradiotherapy for rectal cancer. N Engl J Med 2004;351:1731-40.

5. Sauer R, Liersch T, Merkel S, et al. Preoperative versus postoperative chemoradiotherapy for locally advanced rectal cancer: results of the German CAO/ARO/AIO-94 randomized phase III trial after a median follow-up of 11 years. J Clin Oncol
2012;30:1926-33.

6. Bosset JF, Collette L, Calais $G$, et al. Chemotherapy with preoperative radiotherapy in rectal cancer. N Engl J Med 2006; 355:1114-23.

7. Bujko K, Nowacki MP, Nasierowska-Guttmejer A, Michalski W, Bebenek M, Kryj M. Long-term results of a randomized trial comparing preoperative short-course radiotherapy with preoperative conventionally fractionated chemoradiation for rectal cancer. Br J Surg 2006;93:1215-23.

8. Glimelius B, Pahlman L, Cervantes A; ESMO Guidelines Working Group. Rectal cancer: ESMO Clinical Practice Guidelines for diagnosis, treatment and follow-up. Ann Oncol 2010;21 Suppl 5:v82-6.

9. Guckenberger M, Saur G, Wehner D, et al. Comparison of preoperative short-course radiotherapy and long-course radiochemotherapy for locally advanced rectal cancer. Strahlenther Onkol 2012;188:551-7.

10. Ngan SY, Burmeister B, Fisher RJ, et al. Randomized trial of short-course radiotherapy versus long-course chemoradiation comparing rates of local recurrence in patients with $T 3$ rectal cancer: Trans-Tasman Radiation Oncology Group trial 01.04. J Clin Oncol 2012;30:3827-33.

11. Peeters KC, Marijnen CA, Nagtegaal ID, et al. The TME trial after a median follow-up of 6 years: increased local control but no survival benefit in irradiated patients with resectable rectal carcinoma. Ann Surg 2007;246:693-701.

12. Roh MS, Colangelo LH, O'Connell MJ, et al. Preoperative multimodality therapy improves disease-free survival in patients with carcinoma of the rectum: NSABP R-03. J Clin Oncol 2009;27:5124-30.

13. Sebag-Montefiore D, Stephens RJ, Steele R, et al. Preoperative radiotherapy versus selective postoperative chemoradiotherapy in patients with rectal cancer (MRC CRO7 and NCIC-CTG C016): a multicentre, randomised trial. Lancet 2009;373:811-20.

14. van Gijn W, Marijnen CA, Nagtegaal ID, et al. Preoperative radiotherapy combined with total mesorectal excision for resectable rectal cancer: 12-year follow-up of the multicentre, randomized controlled TME trial. Lancet Oncol 2011;12:57582.

15. Collette $L$, Bosset JF, den Dulk $M$, et al. Patients with curative resection of cT3-4 rectal cancer after preoperative radiotherapy or radiochemotherapy: does anybody benefit from adjuvant fluorouracil-based chemotherapy? A trial of the European Organisation for Research and Treatment of Cancer Radiation Oncology Group. J Clin Oncol 2007;25:4379-86.

16. Mohiuddin M, Marks J, Marks G. Management of rectal cancer: short- vs. long-course preoperative radiation. Int J Radiat Oncol Biol Phys 2008;72:636-43.

17. Peeters $K C$, van de Velde CJ, Leer JW, et al. Late side effects 
of short-course preoperative radiotherapy combined with total mesorectal excision for rectal cancer: increased bowel dysfunction in irradiated patients. A Dutch Colorectal Cancer Group study. J Clin Oncol 2005;23:6199-206.

18. Pollack J, Holm T, Cedermark $B$, et al. Late adverse effects of short-course preoperative radiotherapy in rectal cancer. $\mathrm{Br} J$ Surg 2006:93:1519-25.

19. Birgisson H, Pahlman L, Gunnarsson U, Glimelius B; Swedish Rectal Cancer Trial Group. Adverse effects of preoperative radiation therapy for rectal cancer: long-term follow-up of the Swedish Rectal Cancer Trial. J Clin Oncol 2005;23:8697705.

20. Yeo SG, Oh JH, Kim DY, et al. Preoperative short-course concurrent chemoradiation therapy followed by delayed surgery for locally advanced rectal cancer: a phase 2 multicenter study (KROG 10-01). Int J Radiat Oncol Biol Phys 2013;86:34-9.

21. Yoon M, Park SY, Shin D, et al. A new homogeneity index based on statistical analysis of the dose-volume histogram. J Appl Clin Med Phys 2007:8:9-17.

22. Knoos T, Kristensen I, Nilsson P. Volumetric and dosimetric evaluation of radiation treatment plans: radiation conformity index. Int J Radiat Oncol Biol Phys 1998;42:1169-76.

23. Bazan JG, Hara W, Hsu A, et al. Intensity-modulated radiation therapy versus conventional radiation therapy for squamous cell carcinoma of the anal canal. Cancer 2011;117:3342-51.

24. Du XL, Tao J, Sheng XG, et al. Intensity-modulated radiation therapy for advanced cervical cancer: a comparison of dosimetric and clinical outcomes with conventional radiotherapy. Gynecol Oncol 2012;125:151-7.

25. Heron DE, Gerszten K, Selvaraj RN, et al. Conventional 3D conformal versus intensity-modulated radiotherapy for the adjuvant treatment of gynecologic malignancies: a comparative dosimetric study of dose-volume histograms small star, filled. Gynecol Oncol 2003;91:39-45.

26. Luxton G, Hancock SL, Boyer AL. Dosimetry and radiobiologic model comparison of IMRT and 3D conformal radiotherapy in treatment of carcinoma of the prostate. Int J Radiat Oncol Biol Phys 2004;59:267-84.

27. Arbea L, Ramos LI, Martinez-Monge R, Moreno M, Aristu J. Intensity-modulated radiation therapy (IMRT) vs. 3D conformal radiotherapy (3DCRT) in locally advanced rectal cancer (LARC): dosimetric comparison and clinical implications. Radiat Oncol 2010;5:17.

28. Yu M, Lee JH, Jang HS, et al. A comparison of dosimetric parameters between tomotherapy and three-dimensional conformal radiotherapy in rectal cancer. Radiat Oncol 2013;8:181. 